\title{
АНАЛИЗ РЕЗУЛЬТАТА ПРИМЕНЕНИЯ МЕТОДА ГИРУДОТЕРАПИИ ПРИ ЛЕЧЕНИИ ХРОНИЧЕСКОГО АПИКАЛЬНОГО ПЕРИОДОНТИТА
}

\author{
А. И. Абдуллаева 凶, А. Г. Притыко, П. А. Воронин, Е. Г. Михайлова \\ Российский национальный исследовательский медицинский университет имени Н. И. Пирогова, Москва, Россия
}

\begin{abstract}
Для хронического апикального периодонтита (ХАП) характерно воспаление тканей, окружающих верхушку корня зуба. Отсутствие стабильности результатов стандартного лечения объясняет поиск новых лекарственных средств и методов лечения данного заболевания. Целью исследования было проанализировать влияние гирудотерапии в лечении ХАП при динамическом наблюдении с использованием клинических методов исследования. Пациенты в возрасте от 25 до 40 лет (всего 41 человек) были разделены на основную группу (20 человек) и группу сравнения (21 человек). У всех участников исследования определяли показатели выраженности постоянных болевых ощущений и десневого индекса Gl. B первое посещение показатели выраженности болевых ощущений в группе сравнения $(5,81 \pm 0,65)$ и основной группе $(5,75 \pm 0,92)$ сильно не различались. К третьему посещению у пациентов основной группы боль почти отсутствовала (1,05 \pm 0,34), а в группе сравнения показатели были выше $(4,10 \pm 0,7)$. Результаты клинических исследований указывают на положительное влияние гирудотерапии в составе комплексного лечения.
\end{abstract}

Ключевые слова: гирудотерапия, хронический апикальный периодонтит, воспаление, боль

Вклад авторов: А. И. Абдуллаева - анализ литературы и полученных данных, написание статьи; А. Г. Притыко - анализ полученных данных, участие в написании статьи; П. А. Воронин — обработка полученных данных, статистическая обработка данных, редактирование рукописи; Е. Г. Михайлова обработка полученных данных, редактирование рукописи.

Соблюдение этических стандартов: исследование одобрено этическим комитетом РНИМУ имени Н. И. Пирогова (протокол № 947 от 04 февраля 2019 г.). Все пациенты подписали добровольное информированное согласие на участие в исследовании.

$\triangle$ Для корреспонденции: Айтан Измировна Абдуллаева

ул. Островитянова, д. 1, г. Москва, 117997; aitanka@list.ru

Статья получена: 01.05.2020 Статья принята к печати: 15.05.2020 Опубликована онлайн: 18.05.2020

DOI: $10.24075 /$ vrgmu.2020.028

\section{ANALYSIS OF LEECH THERAPY EFFECTS IN PATIENTS WITH CHRONIC APICAL PERIODONTITIS}

\author{
Abdullaeva Al ${ }^{凶}$, Prityko AG, Voronin PA, Mikhailova EG
}

Pirogov Russian National Research Medical University, Moscow, Russia

Chronic apical periodontitis (CAP) is characterized by tissue inflammation around the tooth tip. Unstable outcomes of current treatments against CAP dictate the need for novel therapeutic techniques and medications. The aim of this study was to analyzed the effects of hirudotherapy on the treatment course in patients with CAP. Forty-one study participants aged 25 to 40 years were divided into the main group (20 patients) and the control group (21 patients). Pain level and the gingival index (Gl) were measured in all study participants. During the first visit, pain scores did not differ significantly between the control (5.81 \pm 0.65$)$ and the main $(5.75 \pm 0.92)$ groups. During the second visit, pain was almost unnoticeable in the main group patients $(1.05 \pm 0.34)$, whereas pain scores were higher in the control group ( $4.10 \pm 0.7)$. Our findings suggest a positive effect of hirudotherapy used in combination with standard treatment regimens.

Keywords: hirudotherapy, chronic apical periodontitis, inflammation, pain

Author contribution: Abdullaeva Al — literature and experimental data analysis, experimental data analysis; Prityko AG — experimental data analysis, experimental data analysis; Voronin PA — statistical analysis, manuscript revision; Mikhailova EG — statistical analysis, manuscript revision.

Compliance with ethical standards: the study was approved by the Ethics Committee of Pirogov Russian National Research Medical University (Protocol № 947 dated February 4, 2019). Informed consent was obtained from all study participants.

$\triangle$ Correspondence should be addressed: Aitan I. Abdullaeva

Ostrovityanova, 1, Moscow, 117997; aitanka@list.ru

Received: 01.05.2020 Accepted: 15.05.2020 Published online: 18.05.2020

DOI: $10.24075 /$ brsmu.2020.028

Распространенность хронического апикального периодонтита (ХАП) в возрасте от 18 лет и старше составляет 95-100\% [1]. Необходимость поиска новых медикаментозных средств и методик лечения этого заболевания обусловлена отсутствием стабильности результатов, получаемых при использовании известных методов лечения. На протяжении веков известен способ лечения пациентов с помощью лекарственных пиявок Hirudo medicinalis - гирудотерапия [2]. В прошлом метод оказался эффективным для лечения ряда заболеваний, включая лечение боевых ран [3]. К абсолютным противопоказаниям относят: онкологические заболевания, нарушения свертываемости крови, тромбоцитопению, непереносимость компонентов слюны пиявок [4, 5]. В настоящее время гирудотерапию используют для облегчения боли, при лечении астмы, гипертонии, мигрени, флебита, варикозного расширения вен, а также стоматологических заболеваний [2, 6-7]. Результаты чаще положительны, но иногда возникают негативные последствия ее использования. Во время укуса пиявки выделяют в рану сложную смесь различных биологически и фармакологически активных веществ, в том числе гирудин [8]. Последний представляет собой полипептид, образующий необратимое соединение с тромбином, что препятствует переходу фибриногена в фиибрин $[9,10]$. Тем самым он улучшает микроциркуляцию в тканях, оказывает противовоспалительное и анальгетическое действия, стимулирует регенерацию тканей [11]. Гирудотерапия одобрена Минздравом России и имеет фармакопейную статью ФС-42-702-97, т. е. Госстандарт лекарственного средства с перечнем показателей и методов контроля качества. В доступной литературе мало работ по оценке эффективности этого метода в лечении периодонтита, и вопрос об обоснованности его использования при ХАП остается до конца нерешенным [12]. 
Таблица 1. Характеристика групп по возрасту и полу

\begin{tabular}{|l|c|c|}
\hline & Основная группа $(n=20)$ & Группа сравнения $(n=21)$ \\
\hline Пол М/Ж & $8 / 12$ & $9 / 12$ \\
\hline Средний возраст (лет), $\mathrm{M} \pm m$ & $31,4 \pm 0,4$ & $30,2 \pm 0,3$ \\
\hline
\end{tabular}

Целью исследования было изучить влияние гирудотерапии в комплексном лечении ХАП на скорость улучшения клинических показателей.

\section{ПАЦИЕНТЫ И МЕТОДЫ}

В январе-марте 2020 г. на базе Научно-практического центра специализированной медицинской помощи детям имени В. Ф. Войно-Ясенецкого были проведены лечение и клинические обследования 41 пациента с ХАП в стадии обострения. Среди обследуемых были 17 мужчин и 24 женщины 25-40 лет. Средний возраст женщин составил $29,1 \pm 1,7$ лет, мужчин 34,6 \pm 1,4 лет. Критерии включения пациентов в исследование: наличие ХАП; повторное эндодонтическое лечение; отсутствие свища, возраст 25-40 лет. Критерии исключения: наличие сопутствующих заболеваний в стадии декомпенсации, обострение хронической сопутствующей патологии, наличие воспалительных заболеваний пародонта, беременность на любом сроке, ВИЧ-инфекция, нарушения в свертывающей системе крови, наличие злокачественных новообразований, отказ больного от участия в исследовании. Диагноз «хронический апикальный периодонтит» (код МКБ: К04.5) ставили на основании данных компьютерной томографии (GENORAY; Ю. Корея).

Все пациенты были разделены на две группы: основную и группу сравнения. В основную группу включены 20 пациентов, В их лечении применяли гирудотерапию в комплексе с принятым в клинике стандартным протоколом лечения данной патологии. Средний возраст женщин составил 30,0 \pm 1,0 год; мужчин - 33,5 \pm 1,3 года. Группа сравнения включила 21 пациента, проходившего лечение без применения гирудотерапии. Средний возраст женщин был 31,5 \pm 1,2 года; мужчин - 28,4 \pm 1,4 года (табл. 1). Между группами отсутствовали значимые различия по полу и возрасту.

Эндодонтическое лечение проводили по стандартам, утвержденным Европейской эндодонтической ассоциацией в 1994 г. (подготовка к эндодонтическому лечению, формирование доступа, установка коффердама, измерение полной рабочей длины, очистка и формирование корневых каналов, пломбирование корневых каналов). У всех пациентов, участвующих в данном исследовании, применяли идентичные препараты и материалы. После эндодонтических манипуляций проводили сеанс гирудотерапии: в область проекции верхушки корня причинного зуба аспирационным методом осуществляли постановку медицинской пиявки на слизистую оболочку альвеолярного отростка до полного кровенасыщения (20-30 мин). Все пиявки были приобретены в ООО
«ГирудоЦентр» (Россия) с сертификатом соответствия POCC RU.АД77.Н00310 (срок действия с 29.01.2018 по 28.01.2021). Сеансы проводили 3 раза: на 1-й, 4-й, 7-й дни с начала лечения, согласно методическим рекомендациям по применению гирудотерапии [13].

Для оценки эффективности лечения определяли показатели выраженности постоянных болевых ощущений и десневого индекса Gl по Loe и Silness (1963) причинного зуба у пациентов с ХАП в группе сравнения и в основной группе в динамике [8]. При оценке болевых ощущений применяли визуальную аналоговую шкалу боли (VAS), где «0» - нет боли; «1-3» - слабая боль; «4» - умеренная боль; «5-6» - сильная боль; «7-9» - очень сильная боль и «10» - нестерпимая боль. Десневой индекс Gl оценивает состояние периодонта по клиническим признакам воспаления десны - гиперемии, отечности и кровоточивости после прикосновения атравматичным зондом, в нашем случае, в области исследуемого зуба. Состояние каждого участка десны оценивают следующим образом: 0 - десна без признаков воспаления; 1 небольшое изменение цвета, легкая отечность, нет кровоточивости при исследовании (легкое воспаление); 2 - покраснение, отек, кровоточивость при исследовании (умеренное воспаление); 3 - выраженная гиперемия, отек, изъязвления, тенденция к спонтанным кровотечениям (тяжелое воспаление) [8].

Статистическую обработку результатов проводили при помощи программного обеспечения: SPSS 21 (IBM SPSS Statistics; США). Значимым уровнем достоверности считали 95\% ( $p \leq 0,05)$.

\section{РЕЗУЛЬТАТЫ ИССЛЕДОВАНИЯ}

У пациентов с ХАП в 1-й день показатели выраженности болевых ощущений значимо не различались. Во второе посещение в основной группе показатели выраженности постоянной боли уменьшились; в третье посещение боль почти отсутствовала и отмечалось противоболевое действие гирудотерапии (табл. 2). На 4-й день в группе сравнения показатели выраженности боли тоже уменьшались, а уже в третье посещение они были значительно выше, чем в основной группе, где применяли гирудотерапию. Небольшое увеличение показателей выраженности болевых ощущений в группе сравнения на 7-й день связано с нормальной реакцией на лечебные манипуляции.

Значения десневого индекса Gl c каждым посещением стабильно снижались в обеих группах, но в основной группе показатели индекса на 4-й и 7-й дни были ниже, чем в группе сравнения. Вместе с анальгезирующим

Таблица 2. Показатели десневого индекса GI и болевого синдрома при ХАП у пациентов основной группы и группы сравнения в динамике

\begin{tabular}{|c|c|c|c|c|c|c|}
\hline & \multicolumn{2}{|c|}{ 1-й день } & \multicolumn{2}{|c|}{ 4-й день } & \multicolumn{2}{|c|}{ 7-й день } \\
\hline Показатели & Основная группа & Группа сравнения & Основная группа & Группа сравнения & Основная группа & Группа сравнения \\
\hline $\begin{array}{l}\text { Болевые ощущения, } \\
\text { баллы }(\mathrm{M} \pm m)\end{array}$ & $5,75 \pm 0,92$ & $5,81 \pm 0,65$ & $2,5 \pm 0,54$ & $3,29 \pm 0,68$ & $1,05 \pm 0,34^{*}$ & $4,1 \pm 0,75$ \\
\hline $\begin{array}{l}\text { Десневой индекс Gl, } \\
\text { баллы }(\mathrm{M} \pm m)\end{array}$ & $1,88 \pm 0,06$ & $1,92 \pm 0,05$ & $1,13 \pm 0,10$ & $1,45 \pm 0,13$ & $0,38 \pm 0,06^{\star}$ & $1,13 \pm 0,06$ \\
\hline
\end{tabular}

Примечание: * $-p<0,05$ по сравнению с группой сравнения. 
эффектом гирудотерапия оказывала противоотечное действие, а также приводила к уменьшению гиперемии

Таким образом, оценка показателей клинических проявлений свидетельствует о положительном влиянии гирудотерапии на комплексное лечение хронического верхушечного периодонтита.

\section{ОБСУЖДЕНИЕ РЕЗУЛЬТАТОВ}

В исследовании показано положительное влияние гирудотерапии в составе комплексного лечения ХАП, что наряду с данными других отечественных исследований еще раз доказывает анальгетический и противовоспалительный эфректы гирудотерапии. В работе, которая согласуется с нашим исследованием, говорится о том, что применение гирудотерапии при деструктивных формах верхушечного периодонтита приводит $\mathrm{K}$ снижению интоксикации в зубочелюстном сегменте, где имеется патологический очаг [14]. В данном исследовании мы показали эффективность гирудотерапии еще и в уменьшении показателей воспалительных изменений тканей периодонта. Так, к третьему посещению в основной группе признаки гиперемии исчезли, при этом в группе сравнения они практически не изменялись на протяжении всего наблюдения. В диссертационной работе 2003 г., которая также согласуется с результатами данного исследования, говорится о том, что использование в комплексном лечении гирудотерапии позволяет в более ранние сроки купировать болевой, отечный, воспалительный синдром и сократить сроки лечения [15]. В нашем исследовании использованы дополнительные параметры в виде оценки болевых ощущений по 10-балльной шкале и определения десневого индекса Gl.

На основании определения показателей постоянной боли, отека и гиперемии на 1-е, 4-е и 7-е сутки комплексного лечения, можно сделать вывод, что данные показатели были ниже у пациентов, в комплекс терапии которых входила гирудотерапия. У больных основной группы отмечалось клиническое улучшение состояния благодаря быстрому разрешению воспалительного процесса и выраженному деконгестивному и противоболевому действиям секрета медицинской пиявки. Побочных явлений при применении гирудотерапии отмечено не было.

\section{ВЫВОДЫ}

Таким образом, на основании результатов проведенного исследования гирудотерапию можно рекомендовать в комплексном лечении ХАП. Полученные результаты могут пригодиться в клинической практике врачастоматолога для более быстрого купирования боли и уменьшения воспаления у пациентов. Несомненно, требуются дополнительные исследования в отдаленные сроки наблюдений с использованием информативных, современных методов диагностики, в том числе рентгенологических.

\section{Литература}

1. Березин К. А., Греков А. Х., Зарипова Э. М., Старцева Е. Ю. Статистические аспекты изучения распространенности хронического апикального периодонтита у взрослого населения. Современные проблемы науки и образования. 2015; 2 (1). URL: http://www.science-education.ru/ry/article/ view?id=19306 (дата обращения: 8.05.2020).

2. Kunal J, Aarti G, Ridhi N, Sunanda D. Hirudotherapy in Medicine and Dentistry. J Clin Diagn Res. 2015; 9 (12): ZE05-ZE07.

3. Сашкина Т. И., Абдуллаева А. И., Рунова Г. С., Салдусова И. В., Зайченко О. В., Фасхутдинов Д. К. и др. Гирудотерапия В лечении хронического генерализованного пародонтита. Вестник РГМУ. 2019; 4: 83-86.

4. Spear M. Medicinal Leech Therapy: Friend or Foe. Plast Surg Nurs. 2016; 36 (3): 121-5.

5. Liu C, Barkley TW Jr. Medicinal leech therapy: New life for an ancient treatment. Nursing. 2015; 45 (11): 25-30.

6. Федотова Ю. М., Костюкова Ю. И. Гирудотерапия: теория и практика. Научное обозрение. Медицинские науки. 2017; 2: 22-25.

7. Kulbida R, Mathes A, Loeser J. Beneficial effects of hirudotherapy in a chronic case of complex regional pain syndrome. J Integr Med. 2019; 17 (5): 383-6.

\section{References}

1. Berezin KA, Grekov AH, Zaripova YeM, Starceva EYu. Statisticheskie aspekty izuchenija rasprostranennosti hronicheskogo apikal'nogo periodontita u vzroslogo naselenija. Sovremennye problemy nauki i obrazovanija. 2015; 2 (1). URL: http://www.science-education.ru/ ry/article/view?id=19306 (data obrashhenija: 8.05.2020). Russian.

2. Kunal J, Aarti G, Ridhi N, Sunanda D. Hirudotherapy in Medicine and Dentistry. J Clin Diagn Res. 2015; 9 (12): ZE05-ZE07.

3. Sashkina TI, Abdullaeva Al, Runova GS, Saldusova IV, Zajchenko OV, Fashutdinov DK, et al. Hirudotherapy in treatment of chronic
8. Şenel E, Taylan Özkan A, Mumcuoglu KY. Scientometric analysis of medicinal leech therapy. J Ayurveda Integr Med. 2019: S09759476(18)30349-8. DOI: 10.1016/j.jaim.2018.11.006.

9. Liu C, Barkley TW Jr. Medicinal leech therapy: New life for an ancient treatment. Nursing. 2015; 45 (11): 25-30.

10. Sig AK, Guney M, Uskudar Guclu A, Ozmen E. Medicinal leech therapy-an overall perspective. Integr Med Res. 2017; 6 (4): 337-43.

11. Kim KS, Sim HS, Shin JH, Hwang JH, Lee SY. The Relationship between Explanation and Patient Compliance in Hirudotherapy. Arch Craniofac Surg. 2017; 18 (3): 179-85.

12. Kruer RM, Barton CA, Roberti G, Gilbert B, McMillian WD. Antimicrobial prophylaxis during Hirudo medicinalis therapy: a multicenter study. J Reconstr Microsurg. 2015; 31 (3): 205-9.

13. Использование метода гирудотерапии в практическом здравоохранении. Методические рекомендации № 2002/78 (утв. Минздравом РФ 15.07.2002). Доступно по ссылке: https://www.lawmix.ru/medlaw/26582.

14. Орлова Е. Е. Гирудотерапия деструктивных форм верхушечного периодонтита [диссертация]. М., 2003.

15. Денискина Е. В. Клинико-лабораторное обоснование гирудотерапии в комплексном лечении хронического периодонтита [диссертация]. М., 2003. generalised periodontitis. Vestnik RGMU. 2019; 4: 79-83.

4. Spear M. Medicinal Leech Therapy: Friend or Foe. Plast Surg Nurs. 2016; 36 (3): 121-5.

5. Liu C, Barkley TW Jr. Medicinal leech therapy: New life for an ancient treatment. Nursing. 2015; 45 (11): 25-30.

6. Fedotova YuM, Kostjukova Yul. Girudoterapija: teorija i praktika. Nauchnoe obozrenie. Medicinskie nauki. 2017; 2: 22-25. Russian.

7. Kulbida R, Mathes A, Loeser J. Beneficial effects of hirudotherapy 
in a chronic case of complex regional pain syndrome. J Integr Med. 2019; 17 (5): 383-6.

8. Şenel E, Taylan Özkan A, Mumcuoglu KY. Scientometric analysis of medicinal leech therapy. J Ayurveda Integr Med. 2019: S09759476(18)30349-8. DOl: 10.1016/j.jaim.2018.11.006.

9. Liu C, Barkley TW Jr. Medicinal leech therapy: New life for an ancient treatment. Nursing. 2015; 45 (11): 25-30.

10. Sig AK, Guney M, Uskudar Guclu A, Ozmen E. Medicinal leech therapy-an overall perspective. Integr Med Res. 2017; 6 (4): 337-43.

11. Kim KS, Sim HS, Shin JH, Hwang JH, Lee SY. The Relationship between Explanation and Patient Compliance in Hirudotherapy. Arch Craniofac Surg. 2017; 18 (3): 179-85.
12. Kruer RM, Barton CA, Roberti G, Gilbert B, McMillian WD. Antimicrobial prophylaxis during Hirudo medicinalis therapy: a multicenter study. J Reconstr Microsurg. 2015; 31 (3): 205-9.

13. Ispol'zovanie metoda girudoterapii $v$ prakticheskom zdravoohranenii. Metodicheskie rekomendacii \# 2002/78 (utv. Minzdravom RF 15.07.2002). Dostupno po ssylke: https://www. lawmix.ru/medlaw/26582. Russian.

14. Orlova EE. Girudoterapija destruktivnyh form verhushechnogo periodontita [dissertacija]. M., 2003. Russian.

15. Deniskina EV. Kliniko-laboratornoe obosnovanie girudoterapii v kompleksnom lechenii hronicheskogo periodontita [dissertacija]. M., 2003. Russian. 